\title{
Associação da microbiota intestinal com o transtorno da ansiedade e depressão
}

\author{
Association of intestinal microbiota with anxiety and depression disorder \\ Asociación de microbiota intestinal con trastorno de ansiedad y depresión
}

Recebido: 26/03/2021 | Revisado: 31/03/2021 | Aceito: 06/04/2021 | Publicado: 17/04/2021

\author{
Bruna Myrele Freitas da Silva \\ ORCID: https://orcid.org/0000-0002-7120-7047 \\ Faculdade Estácio de Teresina, Brasil \\ E-mail: freitasbruna904@gmail.com \\ Ana Carolina da Conceição Lima \\ ORCID: https://orcid.org/0000-0002-3128-3010 \\ Faculdade Estácio de Teresina, Brasil \\ E-mail: carollimabolo24@gmail.com \\ Laynne Soares dos Santos \\ ORCID: https://orcid.org/0000-0003-4951-4670 \\ Faculdade Estácio de Teresina, Brasil \\ E-mail: laynnesantos7@gmail.com \\ Andrea Nunes Mendes de Brito \\ ORCID: https://orcid.org/0000-0002-8270-9018 \\ Faculdade Estácio de Teresina, Brasil \\ E-mail: drea.nunes@hotmail.com
}

\begin{abstract}
Resumo
Introdução: Os desgastes psicossociais que as pessoas passam cotidianamente é um fator importante na causa de doenças psíquicas como a depressão e a ansiedade através do estresse. Quando não são devidamente tratadas, tanto pela impossibilidade mental de não conseguir expressar esses problemas, essas doenças podem se manifestar no corpo através de sintomas psicossomáticos. Metodologia: Trata-se de uma revisão integrativa, foi conduzida uma busca nas bases de dados SciElo, LILACS e Pub Med, e foram utilizadas para as bases de dados as seguintes palavras: "microbiota intestinal" "Transtorno depressivo" e "Transtorno de Ansiedade". Os artigos identificados durante a busca nas bases de dados foram selecionados a partir da leitura dos títulos, seguido dos resumos, incluindo 5 artigos no estudo. Resultados: Os estudos foram selecionados de acordo com os autores, o ano de publicação, local de estudo, tipo de estudo, resultados e conclusão. A relação da microbiota intestinal com a saúde humana foi restringida aos mais comuns distúrbios mentais e de comportamento. Considerações Finais: Chegou-se a conclusão de que é importante ressaltar a importância da realização de estudos sobre esta temática, com o objetivo de contribuir na qualidade de vida de pessoas que possuem estes transtornos.
\end{abstract}

Palavras-chave: Microbiota intestinal; Transtorno depressivo; Transtorno de ansiedade; Prebióticos.

\begin{abstract}
Introduction: The psychosocial stress that people experience daily is an important factor in the cause of mental illnesses such as depression and anxiety through stress. When they are not properly treated, due to the mental impossibility of not being able to express these problems, these diseases can manifest in the body through psychosomatic symptoms. Methodology: This is an integrative review, a search was conducted in the SciElo, LILACS and Pub Med databases, and the following words were used for the databases: "intestinal microbiota" "Depressive disorder" and "Anxiety Disorder". The articles identified during the search in the databases were selected from the reading of the titles, followed by the abstracts, including 5 articles in the study. Results: The studies were selected according to the authors, the year of publication, place of study, type of study, results and conclusion. The relationship between intestinal microbiota and human health has been restricted to the most common mental and behavioral disorders. Final Considerations: It was concluded that it is important to emphasize the importance of conducting studies on this topic, with the aim of contributing to the quality of life of people who have these disorders.
\end{abstract}

Keywords: Intestinal microbiota; Depressive disorder; Anxiety disorder; Prebiotics.

\section{Resumen}

Introducción: El estrés psicosocial que las personas experimentan a diario es un factor importante en la causa de enfermedades mentales como la depresión y la ansiedad por estrés. Cuando no se tratan adecuadamente, debido a la imposibilidad mental de no poder expresar estos problemas, estas enfermedades pueden manifestarse en el organismo a través de síntomas psicosomáticos. Metodología: Se trata de una revisión integradora, se realizó una búsqueda en las bases de datos SciElo, LILACS y Pub Med, y se utilizaron las siguientes palabras para las bases de datos: "microbiota intestinal" "Trastorno depresivo" y “Trastorno de ansiedad". Los artículos identificados durante la búsqueda en las bases 
de datos se seleccionaron a partir de la lectura de los títulos, seguidos de los resúmenes, incluyendo 5 artículos del estudio. Resultados: Los estudios fueron seleccionados según los autores, año de publicación, lugar de estudio, tipo de estudio, resultados y conclusión. La relación entre la microbiota intestinal y la salud humana se ha restringido a los trastornos mentales y del comportamiento más comunes. Consideraciones finales: Se concluyó que es importante destacar la importancia de realizar estudios sobre este tema, con el objetivo de contribuir a la calidad de vida de las personas que padecen estos trastornos.

Palabras clave: Microbiota intestinal; Desorden depresivo; Trastorno de ansiedad; Prebióticos.

\section{Introduçãa}

Atualmente, pesquisas tem enfatizado a possível relação da microbiota gastrointestinal com o desenvolvimento de diversas doenças como obesidade, diabetes, inflamações e, recentemente, em distúrbios mentais e de comportamento como depressão, ansiedade e autismo. Essa conexão entre o intestino e o cérebro e suas vias conhecidas como eixo intestino - cérebro, inclui cérebro, glândulas, intestino, células que participam do sistema imune a microbiota do trato gastrointestinal (Kawashita, 2018)

A depressão é caracterizada pelo baixo humor, pela baixa autoestima e pela perda de interesse em atividades normalmente agradáveis. Já ansiedade é descrita como uma forma comum de transtorno de humor, com fisiopatologia nervosa, endócrina e imunológica (Grochowska, 2018)

Com isso, essa microbiota vem emergindo como um potencial modulador do comportamento do ser humano e seu papel na recuperação ou manutenção da saúde do indivíduo, buscando-se o equilíbrio do microbioma, com a introdução de prebióticos e probióticos na alimentação, dessa forma é reconhecido que esses microrganismos estão envolvidos no desenvolvimento e funcionamento de diversos processos fisiológicos, como digestão, crescimento e manutenção da homeostase (Kawashita, 2018; Rieder, et al., 2017)

Nessa perspectiva, a Nutrição possibilita adequar a alimentação do indivíduo ao seu estado nutricional e, assim, trazer o equilíbrio ao organismo através da alimentação. Vários efeitos de alimentação inadequada sobre a estrutura e função neural têm sido descritos em seres humanos. A sinalização bidirecional entre o trato gastrointestinal e o cérebro é regulada nos níveis neurais, hormonais e imunológicos. Essa construção é conhecida como o eixo cérebro - intestino e é vital para manter a homeostase. A colonização bacteriana do intestino desempenha um papel importante no desenvolvimento pós-natal e na maturação dos sistemas imunes e endócrinos. Esses processos são fatores que sustentam a sinalização do sistema nervoso central (Furtado, et al., 2018)

A dieta influi nas mudanças da microbiota, que age diretamente nos mecanismos ligados ao sistema límbico. Essas áreas encefálicas são principais reguladores de humor, através de estímulos para liberação de hormônios estimulantes dessas regiões centrais. $\mathrm{O}$ estudo mostra que uma dieta com alta ingestão de gordura está mais relacionada com o estresse e sintomas de ansiedade. Assim, como a deficiência de vitaminas pode estar relacionada com o desenvolvimento de transtornos, baixos níveis de vitaminas B2, B6 e B9, além da hipovitaminose (Franca, 2019)

Por outro lado, os desgastes psicossociais que as pessoas passam cotidianamente é um fator importante na causa de doenças psíquicas como a depressão e a ansiedade através do estresse. Quando não são devidamente tratadas, tanto pela impossibilidade mental de não conseguir expressar esses problemas, essas doenças podem se manifestar no corpo através de sintomas psicossomáticos. Assim, o estudo sugere que a adoção de uma dieta saudável, a prática de exercícios físicos diários e intervenções com probióticos pode ser uma boa estratégia para recuperar a microbiota intestinal e melhorar os sintomas da depressão e humor (Medeiros, 2019)

Diante desse e de outros problemas relacionados a microbiota intestinal, surgiu então o questionamento: Como a microbiota está relacionada com transtornos de ansiedade e depressão e, quais as condutas nutricionais devem ser utilizadas para que esse problema seja resolvido? Dessa maneira, este estudo objetiva investigar a relação entre microbiota intestinal e 
transtornos de ansiedade e depressão.

\section{Metodologia}

Trata-se de uma revisão integrativa, realizada a partir de uma abordagem de pesquisa qualitativa, que seguiu as etapas de identificação do tema e questão de pesquisa.

A questão focal "Existe associação da microbiota com os transtornos de ansiedade e depressão?" foi estruturada no formato do acrônimo PICo em que cada letra equivale a um componente da pergunta condutora: P- Adultos I- Microbiota Intestinal, Co- Transtornos de depressão e ansiedade.

Foram adotados como critérios de elegibilidade: estudos observacionais de coorte, caso-controle e estudos transversais que relacionavam microbiota intestinal, transtornos de ansiedade e depressão publicados nos últimos cinco anos em portugues e ingles. Foram excluídos da artigos de revisão, relatos de casos ou séries de casos, cartas ao editor e artigos não ligados ao tema.

Após a determinação da questão focal, foi conduzida uma busca nas bases de dados Scientific eletronic library Online (Scielo), Literatura Latino-americana e do Caribe em Ciências da Saúde (LILACS) e Pub Med. Os descritores da pesquisa foram referenciados nos Descritores em Ciências da Saúde (DeCS) e foram utilizadas para as bases de dados as seguintes palavras: "microbiota intestinal" "Transtorno depressivo" e "Transtorno de Ansiedade". As palavras-chave foram conectadas pelos operadores booleanos AND, OR e NOT. Dessa forma, foi elaborada a seguinte equação de busca "Microbiota intestinal AND Transtorno depressivo OR Depressão", "Microbiota intestinal AND Transtorno de Ansiedade OR Ansiedade" em todas as bases pesquisadas.

Os artigos identificados durante a busca nas bases de dados foram selecionados a partir da leitura dos títulos, seguido dos resumos. Após esta etapa, uma nova avaliação foi realizada para os estudos a serem lidos na íntegra. As referências dos estudos selecionados foram rastreadas, com vistas à inclusão de outros artigos de potencial interesse. O procedimento foi feito por dois pesquisadores simultaneamente e de forma independente, levando em consideração os critérios de inclusão e exclusão pré-definidos. Os trabalhos para os quais houve discordância foram analisados em reunião com os autores para avaliação e consenso sobre a inclusão na revisão. A análise crítica por afinidade de conteúdo das publicações se deu a partir da leitura e interpretação dos conhecimentos. 
Figura1. Fluxograma de seleção de artigos para revisão de literatura.

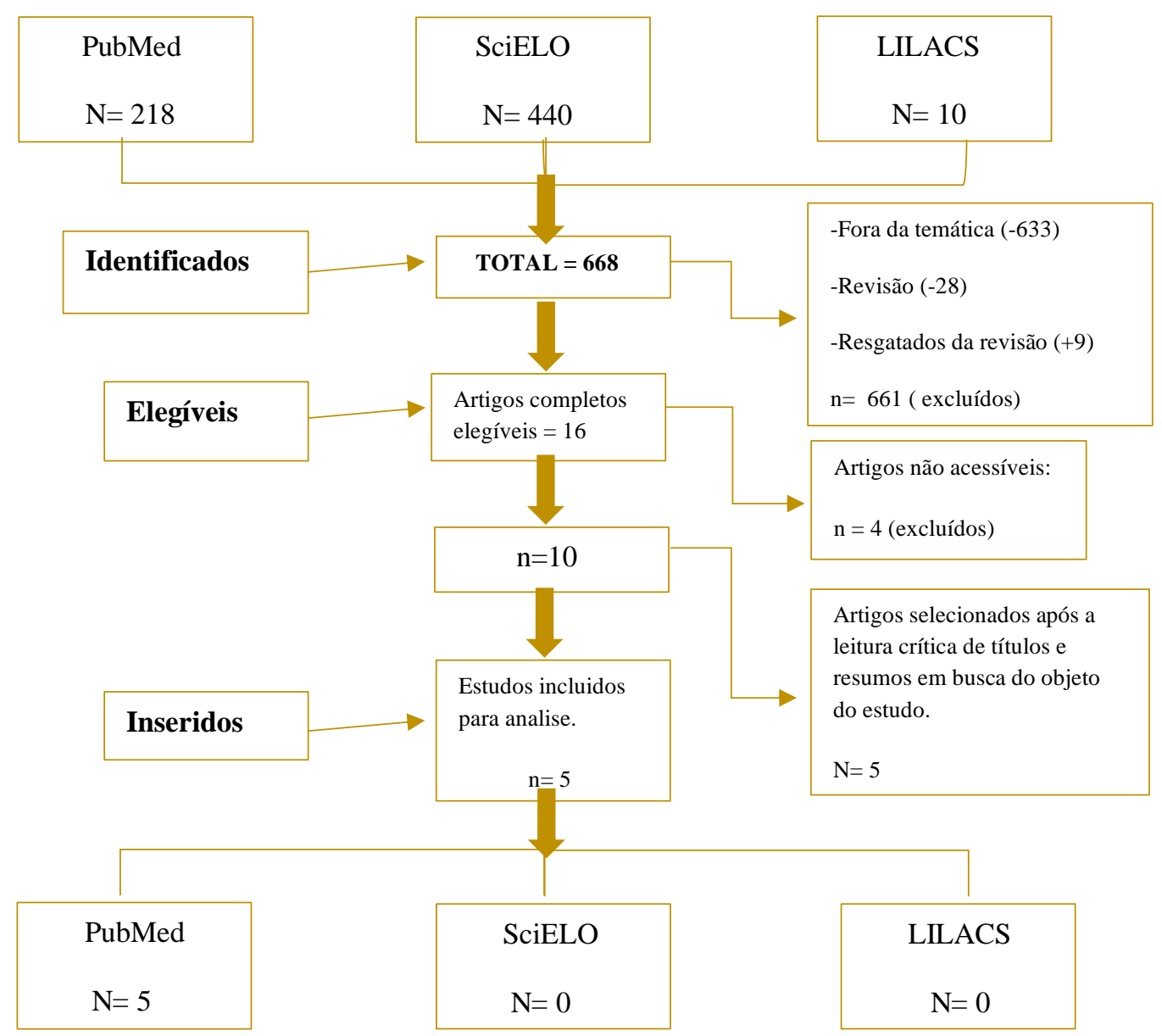

Fonte: Dados da pesquisa (2020).

\section{Resultados e Discussão}

A amostra foi composta por 5 artigos, a base de dados com maior número de artigos selecionados foi a PUBMED. Quanto ao delineamento dos estudos, observou-se que a maior parte era estudos de coortes, conforme o Quadro 1. 
Quadro 1. Caracterização dos estudos relacionando Microbiota intestinal e Transtornos de Ansiedade e Depressão.

\begin{tabular}{|c|c|c|c|c|}
\hline Autor/ano & $\begin{array}{l}\text { Local de } \\
\text { estudo }\end{array}$ & $\begin{array}{l}\text { Tipo de } \\
\text { estudo }\end{array}$ & Resultados & Conclusão \\
\hline $\begin{array}{c}\text { Burokas, et al, } \\
2017\end{array}$ & $\begin{array}{l}\text { Harlan, } \\
\text { Cambridge } \\
\text { shire, } \\
\text { Reino } \\
\text { Unido }\end{array}$ & $\begin{array}{l}\text { Estudo de } \\
\text { Coorte }\end{array}$ & $\begin{array}{l}\text { Administração de prebióticos aumentou acetato } \\
\text { (cecal, propionato e isobutirato), reduzindo } \\
\text { concentrações, mudanças que se correlacionaram } \\
\text { significativamente com os efeitos positivos vistos } \\
\text { no comportamento. Além disso, FOS+GOS } \\
\text { reduziu as elevações induzidas por estresse } \\
\text { crônico nos níveis de corticosterona e citocinas } \\
\text { pró-inflamatórias e comportamento tipo depressão } \\
\text { e ansiedade, além de normalizar os efeitos do } \\
\text { estresse sobre a microbiota. }\end{array}$ & $\begin{array}{l}\text { Sugere fortemente um papel benéfico do } \\
\text { tratamento prebiótico para comportamentos } \\
\text { relacionados ao estresse. Essas descobertas } \\
\text { fortalecem a base de evidências que apoiam o } \\
\text { direcionamento terapêutico do intestino e } \\
\text { microbiota para doenças do eixo cérebro- } \\
\text { intestino, abrindo novos caminhos no campo } \\
\text { da neuropsicofarmacologia nutricional. }\end{array}$ \\
\hline $\begin{array}{l}\text { Morrison, et al, } \\
2016\end{array}$ & Austrália & $\begin{array}{c}\text { Estudo } \\
\text { Transversal }\end{array}$ & $\begin{array}{l}\text { Feito protetor da inibição da caspase- } 1 \text { envolve a } \\
\text { modulação da relação entre o estresse e a } \\
\text { composição da microbiota intestinal, e estabelece } \\
\text { a base para um eixo microbiota intestinal- } \\
\text { inflamassoma-cérebro, pelo qual a microbiota } \\
\text { intestinal via sinalização do inflamassoma modula } \\
\text { as vias que irão alterar a função cerebral e afetar a } \\
\text { depressão e comportamentos semelhantes à } \\
\text { ansiedade. }\end{array}$ & $\begin{array}{l}\text { Redução da bioatividade do inflamassoma } \\
\text { pode representar um viável e estratégia (TDT) } \\
\text { terapêutica direta no tratamento e outros } \\
\text { doenças neuropsiquiátricas com componentes } \\
\text { inflamatórios. Além disso, a modificação } \\
\text { direta da microbiota intestinal pode oferecer } \\
\text { novas oportunidades para atenuar ainda mais } \\
\text { esses distúrbios, por meio de modulação da } \\
\text { sinalização do inflamassoma. }\end{array}$ \\
\hline $\begin{array}{c}\text { Dinan, et al, } \\
2013\end{array}$ & Irlanda & $\begin{array}{l}\text { Estudo de } \\
\text { Coorte }\end{array}$ & $\begin{array}{l}\text { Indicam uma redistribuição das abundâncias } \\
\text { relativas de filos bacterianos dentro da microbiota } \\
\text { intestinal em animais com um fenótipo } \\
\text { relacionado à depressão e ansiedade. No geral, } \\
\text { essas descobertas indicam uma redistribuição das } \\
\text { abundâncias relativas de filos bacterianos dentro } \\
\text { da microbiota intestinal em animais com um } \\
\text { fenótipo relacionado à depressão e ansiedade. }\end{array}$ & $\begin{array}{l}\text { Isso permitirá um entendimento mais completo } \\
\text { da falha de comunicação do eixo cérebro- } \\
\text { intestino em estados de estresse crônico, como } \\
\text { depressão e aqueles pensado para fundamentar } \\
\text { o comum afetivo co-mórbido distúrbios } \\
\text { associados ao IBS. }\end{array}$ \\
\hline $\begin{array}{l}\text { Collins, et al, } \\
2013\end{array}$ & CA, EUA & $\begin{array}{l}\text { Estudo de } \\
\text { Coorte }\end{array}$ & $\begin{array}{l}\text { A administração central do hormônio liberador de } \\
\text { corticotropina (CRH) afeta o comportamento e a } \\
\text { função gastrointestinal. Tratados com CRH } \\
\text { (hormônio liberador de corticotropina) têm níveis } \\
\text { mais altos de comportamento semelhante ao de } \\
\text { ansiedade, conforme demonstrado pelo aumento } \\
\text { da locomoção e da criação em comparação com os } \\
\text { controles tratados com solução salina. }\end{array}$ & $\begin{array}{l}\text { Frequente coexistência de depressão e } \\
\text { distúrbios gastrointestinais funcionais, como o } \\
\text { SII (síndrome do intestino irritável). Achados } \\
\text { sugerem que a depressão comórbida } \\
\text { provavelmente aumenta a gravidade da } \\
\text { disfunção intestinal por meio de alterações } \\
\text { induzidas pela depressão na motilidade } \\
\text { colônica e desestabiliza ainda mais a } \\
\text { microbiota intestinal, resultando na } \\
\text { instabilidade temporal da microbiota } \\
\text { previamente documentada em pacientes com } \\
\text { SII }\end{array}$ \\
\hline $\begin{array}{l}\text { Naseribafrouei, } \\
\text { et al, } 2014\end{array}$ & Noruega & $\begin{array}{c}\text { Estudo } \\
\text { Observacional }\end{array}$ & $\begin{array}{l}\text { Em um baixo nível taxonômico (OTU), no } \\
\text { entanto, não foi possível identificar qualquer OTU } \\
\text { único mostrando correlação significativa com } \\
\text { depressão após correção de descoberta falsa. O } \\
\text { fator de confusão da medicação para depressão } \\
\text { não parecia influenciar o modelo. }\end{array}$ & $\begin{array}{l}\text { Em conclusão, foi encontrado correlações } \\
\text { significativas entre a microbiota intestinal e a } \\
\text { depressão. As correlações, no entanto, eram } \\
\text { complexas com direções opostas para OTUs } \\
\text { intimamente relacionados. }\end{array}$ \\
\hline
\end{tabular}

Fonte: Lima; Silva; Santos (2020).

Burokas, et al, (2017) propõe um papel benéfico do tratamento prebiótico para comportamentos relacionados ao estresse. Essas descobertas fortalecem a base de evidências que apoiam o direcionamento terapêutico do intestino e microbiota para doenças do eixo cérebro-intestino, abrindo novos caminhos no campo da neuropsicofarmacologia nutricional.

Os psicobióticos foram previamente definidos como bactérias vivas (probióticos) que, quando ingeridas, conferem benefícios para a saúde mental através de interações com bactérias intestinais comensais. Como uma classe de probióticos, essas bactérias são capazes de produzir substâncias neuroativas, como o ácido gama-aminobutírico e a serotonina, que atuam no eixo intestino-cérebro (Sarkar, et al., 2016; Dinan, et al, 2013).

Dessa maneira, os probióticos, definidos como microrganismos vivos que, quando ingeridos em quantidades adequadas, exercem um benefício para a saúde no hospedeiro (Dinan, et al., 2011), são entidades transitórias que colonizam o trato gastrointestinal, influenciam várias vias e estão disponíveis como suplemento. Está bem estabelecido que eles geram efeitos 
terapêuticos em muitos distúrbios ligados ao TGI (Parvez, et al., 2006).

Estudos demonstraram que a suplementação de prebióticos reduz a capacidade de resposta ao estresse, ansiedade e comportamento do tipo depressivo, aumenta a expressão do fator neurotrófico derivado do cérebro (BDNF) e melhora a cognição. Em estudos clínicos, a suplementação de prebióticos aumentou os níveis de AGCC, melhorou os sintomas de comportamento social e os padrões de sono em pacientes com transtorno do espectro do autismo (TEA) e reduziu os escores de ansiedade em pacientes com síndrome do intestino irritável. (Barichello, et al,2020; Sarkar, et al., 2016; Dinan, et al, 2013).

Os gêneros primáros dos microrganismos utilizados como probióticos incluem o Bifibbacterium Gram-positivo e os Lactobacilos, que não possuem cadeias de lipopolissacarídeos pró-inflamatórios e, portanto, não estimulam as reações imunológicas. Além disso, a presença de tipos de bactérias Bifidobacterium e Lactobacilos ajudam a instruir o sistema imunológico a distinguir entre bactérias intestinais e anti-inflamatórias, criando assim uma resposta imune apropriada identificando organismos antigênicos (Sudo, et al, 2004).

Nessa lógica, Morrison, et al, (2016) afirma que a redução da bioatividade do inflamassoma pode representar uma viável estratégia terapêutica direta (TDT) no tratamento e outros doenças neuropsiquiátricas com componentes inflamatórios. Além disso, a modificação direta da microbiota intestinal pode oferecer novas oportunidades para atenuar ainda mais esses distúrbios, por meio de modulação da sinalização do inflamassoma.

Assim, o inflamassoma é um complexo multiproteico intracelular que atua na ativação de enzimas da família cisteínaaspartato proteases (CASPASES) como uma estrutura essencial para a regulação da imunidade em condições fisiológicas e no reconhecimento de sinais de perigo a diferentes componentes (Morrison, et al., 2016).

É indicado uma redistribuição das abundâncias relativas de filos bacterianos dentro da microbiota intestinal em animais com um fenótipo relacionado à depressão e ansiedade. No geral, essas descobertas indicam uma redistribuição das abundâncias relativas de filos bacterianos dentro da microbiota intestinal em animais com um fenótipo relacionado à depressão e ansiedade. (Dinan, et al, 2013.)

Existem evidências emergentes de que a composição microbiana (microbiota) do intestino é alterada na síndrome do intestino irritável (SII), mas a base para isso é mal compreendida. Achados indicam que a indução de ansiedade e influencia a composição da comunidade microbiana no e sugerem que essas mudanças são induzidas pelo aumento da ativação da resposta ao estresse e perturbação do habitat microbiano por meio de alterações na motilidade colônica (Park, et al, 2013).

Naseribafrouei, et al., (2014) verificaram correlação entre microbiota e depressão. Não houve diferenças significativas entre pacientes deprimidos e não deprimidos com relação a riqueza de espécies, embora tenha havido uma ligeira tendência. Em conclusão, foi encontrado correlações significativas entre a microbiota intestinal e a depressão. As correlações, no entanto, eram complexas com direções opostas para Unidades Taxonômicas Operacionas (OTU's) intimamente relacionados.

Ainda que tal assunto seja de grande relevância para a sociedade, verificou-se que são escassos os estudos correlacionando à depressão, ansiedade e a microbiota intestinal, especialmente no que se diz respeito à relação microbiota com a presença de transtorno depressivo. Os estudos já realizados permitem a obtenção de informações relevantes quanto à contribuição da microbiota intestinal para o equilíbrio do organismo como um todo, não apenas para o processo digestório como se afirmava em momento anterior. Nesse modo, contém na literatura o estimulo do uso de probióticos para modulação de sintomas gastrintestinais e psíquicos. Espera-se que novos resultados sobre o papel da dieta na síntese de serotonina auxiliem o tratamento para pacientes com quadros depressivos.

\section{Considerações Finais}

Estudos sobre Microbiota Intestinal e Transtornos de Ansiedade e Depressão estão sendo cada vez mais realizados, contudo, ainda são escassas pesquisas que relacionem as duas temáticas. Dessa maneira, é relevante ressaltar a importância da 
realização de mais estudos sobre esta importante temática, a fim de contribuir para o aumento da qualidade de vida de pessoas que possuem estes transtornos.

Verificou-se que pessoas com transtornos de ansiedade e depressão apresentam composição microbiana alterada possivelmente pelo estresse causados pelos transtornos que repercute gerando alterações na mobilidade do colón. Portanto, a nutrição e uma dieta bem equilibrada, rica em nutrientes e probióticos contribuem para reduzir as alterações da microbiota, equilibrando sua composição e melhorando a qualidade de vida de pessoas com transtornos de ansiedade e depressão.

\section{Referências}

Andrade, V. L. A., Anjos, E. M. S., Amorim, N. R., Iskanda, S. M., Moura, M. T. R. M., Oliveira, K. A., Pereira, M. V. R., Pereira, M. R. A., \& Regazzoni, L. A. A. (2015). Obesidade e microbiota intestinal. Revista de Medicina de Minas Gerais, 25(4): 583-589.

Andrade, A., Liz, C. M., Viana, M. S., \& Rentz, A. (2017). Autoestima, imagem corporal e depressão de adolescentes em diferentes estados nutricionais. Revista de saúde publica. 19 (1). doi.org/10.15446/rsap.v19n1.47697

Antunes, A. E. A., Bueno, R. G. A. L., Moraes, A. L. F., \& Rojas, M. F. (2019). Suplementação Com Probióticos E Depressão: Estratégia Terapêutica? Revista De Ciências Médicas, 28(1):31-47. http://dx.doi.org/10.24220/2318-0897v28n1a4455

Ayeta, A. C., Cunha, A. C. B., Heidelmann, S. P. \& Saunders, C. (2015). Fatores nutricionais e psicológicos associados com a ocorrência de picamalácia em gestantes. Rev Bras Ginecol Obstet 37(12):571-7.

Burokas, A. (2017). Visando o eixo microbiota-intestino-cérebro: os prebióticos têm efeitos ansiolíticos e antidepressivos e revertem o impacto do estresse crônico em camundongos. Psiquiatria biológica 82(7): 472-487.

Blennerhassett, P. A., Collins, J., \& Park, A. J. (2013). Função colônica alterada e perfil da microbiota em um modelo de camundongo com depressão crônica. Revista Neurogastroenterol Motil. 25: 733-575.

Costa, A. P. R., Lach, G., Morais, L. H., \& Hoeller, A. A. (2017). Envolvimento da flora intestinal na modulação de doenças psiquiátricas. Revista de Ciências da Saúde. 29 (1): 64-82. 10.14295/vittalle.v29i1.6413

Cavalcante, L. G. C., \& Brito, A. N. M. (2021). Efeitos da suplementação probiótica sobre a obesidade e o Diabetes Mellitus. Rev Interd. 13(1).

Dinan, T. G., \& Cryan, J. F. (2013). Melancholic microbes: a link between gut microbiota and depression? Neurogastroenterology \& Motility, 25 (9): 713-9. $10.1111 / \mathrm{nmo} .12198$.

França, T. B. (2019). Interação entre o eixo microbiota-intestino-cérebro, dieta e transtornos de humor: uma revisão narrativa. Universidade Federal do Pernambuco- Vitória de Santo Antão.

Furtado, C. C., Silva, A. L. B., \& Walfall, A. M (2018). Psicobióticos Uma Ferramenta Para o Tratamento no Transtorno da Ansiedade e Depressão. Revista UNILUS Ensino e Pesquisa. 15 (40): 2318-2083.

Grochowska, M., Wojnar, M., \& Radkowski, M (2018). The gut microbiota in neuropsychiatric disorders. Revista Acta Neurobiol. Exp, 78: 69-81.

Gomesa, D. O. V. S., \& Morais, M. B. (2019). Microbiota intestinal e emprego dos pro bióticos na constipação intestinal em crianças e adolescentes: revisão sistemática. Revista Paulista de Pediatria. 38: 1984 - 0462. https://doi.org/10.1590/1984-0462/2020/38/2018123

Kawashita, R(2018). A influência da microbiota intestinal na saúde humana e apossível relação com transtornos mentais e comportamentais.Trabalho de Conclusão de Curso de FarmáciaBioquímica. Faculdade de Ciências Farmacêuticas-Universidade de São Paulo, São Paulo.

Naseribafrouei, A Hestad, K, Avershina, E, Sekelja, M, Linløkken, A, Wilson, R, Rud, K (2014). Correlação entre a microbiota fecal humana e a depressão. Neurogastroenterol Motil, 26 (8): 1155-62. 10.1111/nmo.12378

Machado, M. C. L. (2017). Influência de Antipsicóticos de Segunda Geração na Composição da Microbiota Intestinal. Dissertação (Mestrado em Medicina Molecular)- UFMG. Belo Horizonte.

Martins, A. L. P. (2018). Perfil da Microbiota residente intestinal e sua relação coma depressão. 2018. Trabalho de Conclusão de Curso (Bacharelado em Nutrição). Centro Universitário de Brasília.

Medeiros, A. C., \& Maynard, D. C. (2019). A influência do microbioma intestinal no desenvolvimento de processos depressivos e o uso de probióticos como tratamento. Trabalho de Conclusão de Curso (Bacharelado em Nutrição). Centro Universitário de Brasília.

Oliveira, B. A. S., \& Protachevicz, A. P. (2019). Multifatoriedade das doenças psiquícas: Asserções acerca do microbioma humano. In: XVII Jornada Científica dos Campos Gerais.

Perandre, Y. H. T., \& Haydu, V. B. (2018). Um programa de intervenção para transtorno de ansiedade social com o uso da realidade virtual. Revista Trends in Psychology. 26 (2):851-866. 10.9788/TP2018.2-12Pt.

Silva, B. P., Silva, R. G., \& Souza, S. M. (2019). Microbiota intestinal e sua relação com o transtorno de ansiedade. rev. conexão eletrônica, 16 ( 1). 